\title{
HSP70 inhibits high glucose-induced Smad3 activation and attenuates epithelial-to-mesenchymal transition of peritoneal mesothelial cells
}

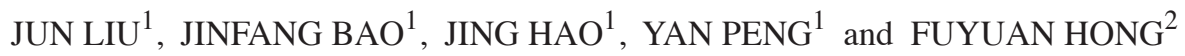 \\ ${ }^{1}$ Department of Nephrology, The First People's Affiliated Hospital, Shanghai Jiao Tong University, Shanghai 200080; \\ ${ }^{2}$ Department of Nephrology, Fujian Provincial Hospital, Fuzhou, Fujian 350001, P.R. China
}

Received September 16, 2013; Accepted April 2, 2014

DOI: $10.3892 / \mathrm{mmr} .2014 .2279$

\begin{abstract}
Heat shock proteins (HSPs) are molecular chaperones that were initially identified as proteins expressed following exposure of cells to environmental stress. However, the function of HSPs in epithelial-to-mesenchymal transition (EMT) of peritoneal mesothelial cells remains unknown. In the present study, the regulation of HSPs and their function in cell EMT, particularly in rat peritoneal mesothelial cells (RPMCs), and the surrounding glucose concentrations and the molecular mechanism involved were investigated. This study explored the effect of HSP70 on high glucose (HG)-induced EMT by overexpression and small interfering RNA (siRNA) knockdown of HSP70, as well as the underlying molecular mechanisms. It was found that HSP70 inhibits HG-induced EMT by modulating Smad expression and activation. HSP70 overexpression inhibited phosphorylation and nuclear translocation of p-Smad3 and p-Smad4, while siRNA of HSP70 enhanced HG-induced Smad3 and Smad4 phosphorylation and EMT. Furthermore, HSP70 suppressed EMT by inhibiting the generation of reactive oxygen species (ROS) induced by HG. In conclusion, HSP70 inhibits EMT of peritoneal mesothelial cells primarily by exerting domain-specific effects on
\end{abstract}

Correspondence to: Dr Fuyang Hong, Department of Nephrology, Fujian Provincial Hospital, 134 Dong Street, Fuzhou, Fujian 350001, P.R. China

E-mail: fuyuan995311@163.com

Abbreviations: RPMCs, rat peritoneal mesothelial cells; HG, high glucose; CAPD, continuous ambulatory peritoneal dialysis; ESRD, end-stage renal disease; DMEM, Dulbecco's modified Eagle's medium; FITC, fluorescein isothiocyanate; RIPA, radioimmunoprecipitation assay; PBS, phosphate-buffered saline; DCF-DA, 2,7-dichlorofluorescein-diacetate; TBS, Tris-buffered saline; PDF, peritoneal dialysis fluid; PI, propidium iodide; EMT, epithelial-to-mesenchymal transition; SMA, smooth muscle actin; HSP70, heat shock protein 70; ROS, reactive oxygen species

Key words: heat shock protein 70, EMT, HG, reactive oxygen species, Smad
Smad3 and Smad4 activation and reducing the release of ROS. HSP70 may be a novel therapeutic target for peritoneal dialysis patients with peritoneal fibrosis.

\section{Introduction}

A rapid increase has occurred in the number of patients with dialysis that are $>65$ years of age (1). Elderly patients are often in poor health and physically incapacitated, susceptible to malnutrition and have multiple complicating medical disorders in addition to end-stage renal disease. Continuous ambulatory peritoneal dialysis (PD) offers numerous advantages to elderly patients, including hemodynamic stability, steady-state chemistries and no requirement to create vascular access. Conventional PD solutions contain glucose as an osmotic agent as it is inexpensive, safe and readily metabolized. However, the use of glucose-based PD fluid may cause a number of side-effects, including dehydration, hypokalemia, hyperglycemia, metabolic alkalosis and chemical peritonitis. Previous studies indicated that high glucose (HG) induces a complex mixture of proinflammatory and profibrotic stimuli during peritoneal epithelial-to-mesenchymal transition (EMT) in vivo $(2,3)$. EMT is a dynamic and complex process that may require the participation of numerous growth factors or cytokines (4). Therefore, factors that regulate EMT are attracting increasing attention as inducers of peritoneal fibrosis.

The expression of heat shock proteins (HSPs) is induced in response to a wide variety of physiological and environmental insults. Their functions as molecular chaperones allow cells to adapt to gradual changes in their environment and to survive in otherwise lethal conditions (5). HSP70, as one of the HSPs, is an ATP-dependent molecular chaperone which performs house-keeping functions; controlling the activity, turnover and trafficking of a variety of proteins, including protein kinases, steroid receptors and transcription factors. HSP70 exhibits an important role in various signaling pathways that have crucial roles in growth control, cell survival and developmental processes $(6,7)$. However, the role of HSP70 in the HG-induced EMT of peritoneal mesothelial cells is unknown. The present study investigated the regulation of HSPs and their role in cell EMT, particularly in rat 
peritoneal mesothelial cells (RPMCs), and the surrounding glucose concentrations and the molecular mechanism involved.

In the present study, whether HG influences HSP expression and to what extent HSPs contribute to HG-induced EMT in RPMCs was investigated.

\section{Material and methods}

Reagents. Penicillin-streptomycin $(5,000 \mathrm{U} / \mathrm{ml}$ penicillin; 5,000 U/ml streptomycin), Dulbecco's modified Eagle's medium (DMEM)/F12 and fetal bovine serum (FBS) were obtained from Gibco-BRL (Grand Island, NY, USA). Triton X-100 and dimethylsulfoxide were purchased from Sigma (St. Louis, MO, USA). HG was obtained from Xinhua Pure Chemical Industries (Shenyang, China). Anti-collagen type I, anti- $\beta$-actin, and anti-phospho Smad 3 and 4 antibodies were obtained from Santa Cruz Biotechnology, Inc. (Santa Cruz, CA, USA); anti- $\alpha$-SMA and anti-vimentin antibodies were purchased from Sigma; and anti-E-cadherin antibody was obtained from BD Biosciences (San Jose, CA, USA). An ECL kit was purchased from Thermo Scientific Pierce (Rockford, IL, USA). A FACSCalibur flow cytometer was purchased from BD Biosciences. All reagents used were trace element analysis grade. All water used was glass distilled.

Isolation and culture of RPMCs. RPMCs were isolated and cultured. Sprague-Dawley rats were purchased from the experimental Animal Breeding Center of Shanghai Jiao Tong University (Shanghai, China). The handling of animals was in accordance with provisions of Medical Ethics. Briefly, surgically resected omenta from Sprague-Dawley rats were digested with $0.125 \%$ trypsin for $25 \mathrm{~min}$ at $37^{\circ} \mathrm{C}$, followed by neutralization with DMEM/F12 medium supplemented with $10 \%$ FBS. The tissue-free cells were then centrifuged at $60 \mathrm{x} \mathrm{g}$ at $4^{\circ} \mathrm{C}$ for $5 \mathrm{~min}$, followed by removal of the supernatant. The cell pellet was resuspended to a final volume of $4 \mathrm{ml}$ in culture medium and then seeded in $25-\mathrm{cm}^{2}$ tissue culture flasks. The culture medium consisted of DMEM/F12 medium supplemented with $10 \% \mathrm{FBS}, 2 \mathrm{mmol} / \mathrm{l} \mathrm{L}$-glutamine, $100 \mathrm{IU} / \mathrm{ml}$ penicillin and $100 \mathrm{mg} / \mathrm{ml}$ streptomycin. After 1-3 days incubation at $37^{\circ} \mathrm{C}$, the media were changed for the first time. Cells were then transferred to serum-free DMEM/F12 medium for overnight starvation prior to each experiment.

Identification of peritoneal mesothelial cells. The RPMCs were observed under a phase contrast inverted microscope and identified using immunocytochemistry. The immunohistochemical antibodies used included vimentin cytokeratin VIII factor-related antigen and leukocyte CD45 antibodies. The cells were seeded and fixed in $10 \%$ neutral formalin for $30 \mathrm{~min}$. Subsequently, antigen retrieval and DAB color dehydration were performed. The cultured cells were observed under a light microscope and cells staining positive for vimentin were characteristic mesothelial cells.

RNA interference plasmid construction and transient transfection. A small interfering RNA (siRNA)-DNA hybrid was formed from DNA corresponding to the rat HSP70 cDNA sequence (5'-AAGGCCAACAAGATCACCAT-3') and an antisense RNA strand [5'-AUGGUGAUCUUGUUGG
CCU(dTdT)-3']. RNA interference plasmid construction was performed by cloning the synthesized oligonucleotide into a pSilencer 2.1-U6-neo plasmid. All these procedures were conducted by Genechem Co. (Shanghai, China). The target sequences of the HSP70 siRNA and control HSP70 siRNA were BLAST (http://blast.ncbi.nlm.nih.gov/Blast.cgi) searched against the GenBank database (https:/www.ncbi.nlm.nih. gov/genbank/). The HSP70 targeting sequence matched exactly with partial sequences of the rat HSP70 gene, but not with any other genes. The control siRNA did not match any known rat gene. Transient transfections were performed using Lipofectamine 2000 (Invitrogen, Carlsbad, CA, USA), according to the manufacturer's instructions. All these experiments were performed in six-well tissue culture plates with cells plated to reach 50-60\% confluence on the day of transfection. The transfection efficiency averaged between 50 and $70 \%$. Cells were allowed to recover in medium for $24 \mathrm{~h}$ after transfection.

HSP70 overexpression plasmid construction and transient transfection. Plasmids expressing a HSP70-cDNA gene plasmid (constructed by Genechem Co., Shanghai, China). were transfected into peritoneal mesothelial cells using Lipofectamine 2000 according to the manufacturer's instructions. All of these experiments were performed in six-well tissue culture plates with cells plated to reach $50-60 \%$ confluence on the day of transfection.

Quantitative polymerase chain reaction ( $q P C R)$. Total RNA from the cultured cells was isolated using TRIzol reagent (Invitrogen). RNase-free DNase I was used to eliminate genomic DNA contamination in the RNA samples. The 260/280 absorbance ratio was measured for verification of the purity of the RNA. The sequences of the HSP70 and $\beta$-actin genes were obtained from the GenBank database, and specific primers for them were designed over an exon-exon junction with Primer Premier, version 5.0 (Premier Biosoft, Palo Alto, CA, USA). The following primer sequences were used: 5'-AGC GGG AAA TCG TCG GTG-3' and 5'-GGG TAC ATG GTG GTG CCG-3' for $\beta$-actin; and 5'-TA CAT ATG GCC AAA GCC GCG GCA GTC G-3' and 5'-TG CTC GAG ATC TAC CTC CTC AAT GGT GGG-3' for HSP70. PCR reactions were performed with a Gene Amp PCR system 9700 (Perkin-Elmer, Waltham, MA, USA) and 35 cycles of amplification were conducted. The amplified products were separated by electrophoresis on a $2 \%$ agarose gel and visualized by ethidium bromide staining. The expression of HSP70 was semi-quantitated using $\beta$-actin as an internal control. Image density was quantified with a FluorImager SI (Amersham Pharmacia Biotech, Amersham, UK).

Western blot analysis. All cells were washed twice with cold PBS and resuspended in five volumes of ice-cold extract buffer $(20 \mathrm{mM}$ western blot analysis Hepes-KOH, $1.5 \mathrm{mM}$ $\mathrm{MgCl}_{2}, 1 \mathrm{mM}$ EDTA, $1 \mathrm{mM}$ ethylene glycol tetraacetic acid, $1 \mathrm{mM}$ dithiothreitol, and $0.1 \mathrm{mM}$ phenylmethanesulfonyl fluoride, $\mathrm{pH} 7.5$ ) for $15 \mathrm{~min}$ at $4^{\circ} \mathrm{C}$. Lysates were centrifuged at $25,000 \mathrm{x} \mathrm{g}$ for $15 \mathrm{~min}$. The protein concentrations of the supernatants were determined using the Micro BCA kit method (Sigma). The samples (10 $\mu \mathrm{g}$ protein) were separated 
A

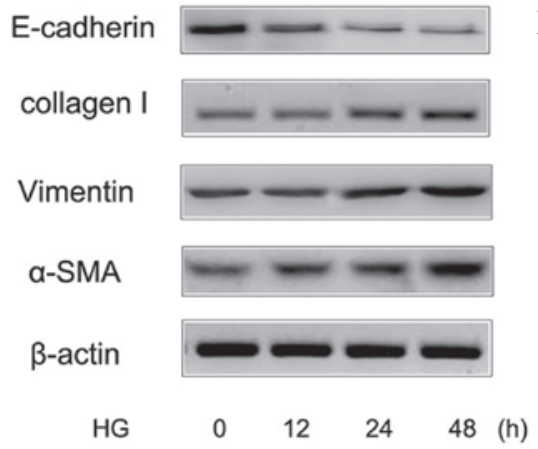

B

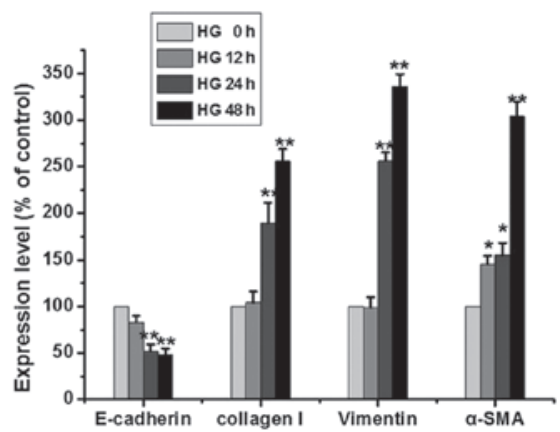

Figure 1. Effect of HG on protein expression of E-cadherin, vimentin, $\alpha$-SMA and collagen I in RPMCs. (A) RPMCs were treated with 25 mM HG at different time points to induce EMT, and the expression of E-cadherin, vimentin, collagen I and $\alpha$-SMA was detected by western blotting. (B) Values represent the mean \pm standard error of the mean of three independent experiments performed. $\beta$-actin was used as the loading control. ${ }^{* *} \mathrm{P}<0.01$ vs. HG $0 \mathrm{~h}$ group, ${ }^{*} \mathrm{P}<0.05$ vs. HG 0 h group. $\alpha$-SMA, $\alpha$-smooth muscle actin; HG, high glucose; RPMCs, rat peritoneal mesothelial cells.

by sodium dodecyl sulfate-polyacrylamide gel electrophoresis (SDS-PAGE) with $10 \%$ and $8 \%$ polyacrylamide gel. The primary antibodies used included anti-collagen type I (1:250), anti- $\alpha$-SMA (1:400), anti- $\beta$-actin (1:400), anti-vimentin (1:400), anti-E-cadherin (1:400), anti-phospho Smad 3 (1:800) and anti-phospho Smad 4 (1:800). These separated proteins were electrotransferred to a Hybond-polyvinylidenefluoride (PVDF) membrane. The individual SDS gel was distinguished by placing the protein molecular weight marker (Invitrogen) in a different but consistent position. The PVDF membrane was then soaked in a blocking solution [5\% nonfat milk in Tris-buffered saline and Tween 20 (TBST) buffer $(20 \mathrm{mM}$ Tris-HCl, pH 7.5, 0.5 M NaCl, 0.1\% Tween 20)] for $2 \mathrm{~h}$ at room temperature. The soaked PVDF membrane was then incubated in TBST containing primary antibodies overnight at $4^{\circ} \mathrm{C}$, washed with TBST buffer three times for 5 min each, and incubated at room temperature for $2 \mathrm{~h}$ in TBST containing horseradish peroxidase (HRP)-conjugated goat anti-mouse and goat anti-rabbit IgG antibodies (Santa Cruz Biotechnology, Inc.). The membrane was washed with TBST buffer three times for $10 \mathrm{~min}$ each. The membranes were incubated in ECL reagent (Thermo Scientific Pierce) for HRP (30 sec) and exposed to autoradiography film for visualization of the bands. The relative amounts of various proteins were analyzed. The results were quantified by Quantity One Software (Bio-Rad, Hercules, CA, USA).

Detection of intracellular reactive oxygen species (ROS) level. To determine the levels of ROS generation within HG-treated cells, fluorescence-activated cell sorting analysis was performed. Cells were stained with $5 \mu \mathrm{g} / \mathrm{ml}$ dihydrodichlorofluorescein diacetate (DCF-DA) for $30 \mathrm{~min}$, subjected to flow cytometry using a FACSCalibur and analyzed by CellQuest software (BD Biosciences, San Jose, CA, USA).

Statistical analysis. Statistical analysis was performed using SPSS software (version 18.0, SPSS, Inc., Chicago, IL, USA). Data are expressed as the mean \pm standard error of the mean. Variance was homogenous for use of standard analysis of variance (ANOVA) methodology. After statistical significance was established by ANOVA, individual comparisons were made using Tukey's multiple comparison test. $\mathrm{P}<0.05$ was considered to indicate a statistically significant difference.

\section{Results}

Effect of HG on EMT of peritoneal mesothelial cells. RPMCs were isolated and cultured. The RPMCs were positive for cytokeratin and vimentin and negative for the VIII factorrelated antigen and leukocyte CD45 antigen. To elucidate the mechanism of EMT by HG, the expression levels of E-cadherin, vimentin, collagen I and $\alpha$-smooth muscle actin (SMA) were monitored. Exposure of peritoneal mesothelial cells to HG (25 mM glucose) for $0-48 \mathrm{~h}$ reduced the protein expression levels of E-cadherin and increased the protein expression levels of the vimentin, collagen I and $\alpha$-SMA over time (Fig. 1). Using western blotting, it was shown that there was time-dependent change in the expression levels of EMT-related proteins in HG-treated peritoneal mesothelial cells. These results indicated that HG could induce EMT and lead to increased EMT events in peritoneal mesothelial cells.

Effect of HSP70 on HG-induced EMT of peritoneal mesothelial cells. HSP70, as a ubiquitously expressed protein, is upregulated by variable stresses, including heat, oxidative stress and chemical injury in the cells (8). Therefore, whether the treatment of peritoneal mesothelial cells with HG changes the expression of HSP in the process was considered in the present study. Cells were treated with $25 \mathrm{mM}$ glucose for 0-48 h, and the expression of HSP70 was determined by western blotting. As shown by Fig. 2A and B, HG enhanced the levels of HSP70, with induction sustained upon HG stimulation compared to the control. To determine the role of HSP70, the effects of HSP70 were studied by modulating HSP70 expression through siRNA knockdown and overexpression. qPCR analysis was performed on isolated total RNA to determine the levels of HSP70 mRNA following transfection of peritoneal mesothelial cells with HSP70 siRNA and HSP70 overexpression plasmid for different time periods (Fig. 2C and D). From the results of the qPCR, it was found that siRNA knockdown and HSP70 overexpression were the most efficient following transfection for $48 \mathrm{~h}$, and the inhibition rate of siRNA knockdown reached $>50 \%$ compared with the levels of the control. Control, HSP70-siRNA knockdown and HSP70 overexpression cells were treated with $25 \mathrm{mM}$ glucose, and the expression levels of E-cadherin, vimentin, collagen I and $\alpha$-SMA were detected by western blot analysis (Fig. $2 \mathrm{E}$ 
E

A

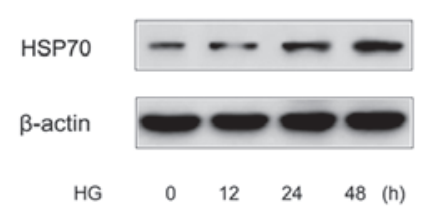

B

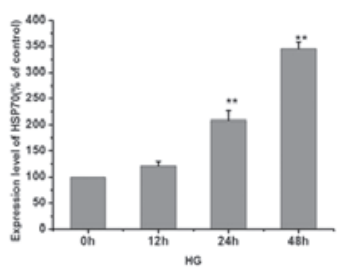

D

HSP7OMRNA

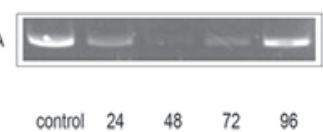

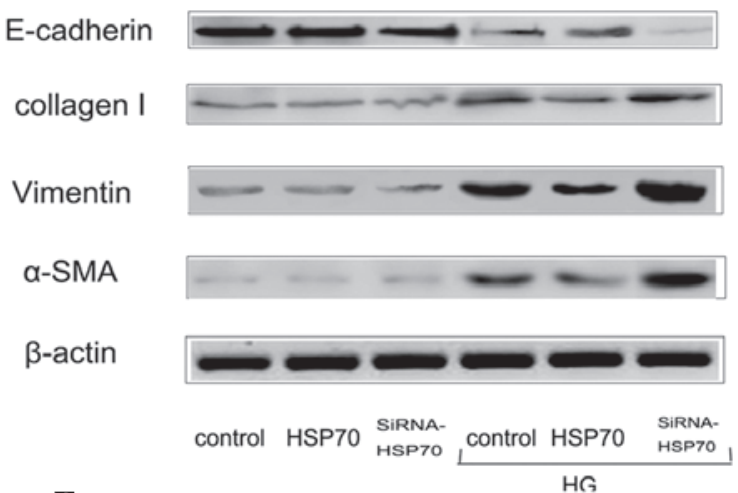

F

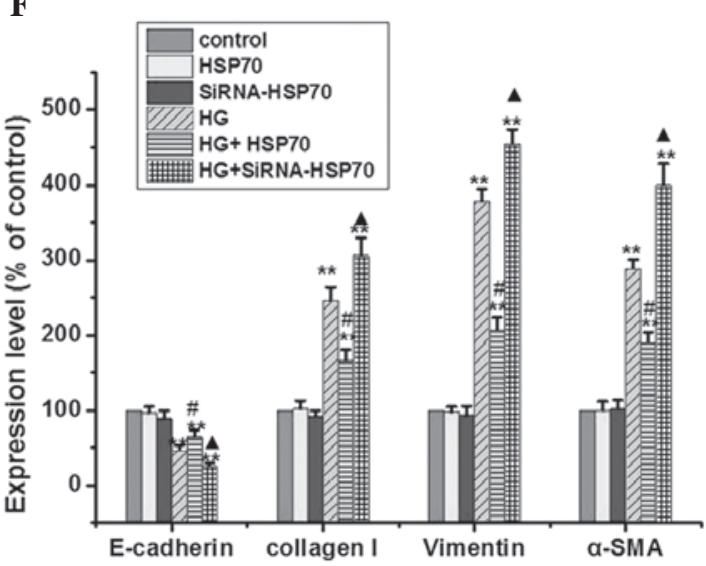

Figure 2. Effects of transfection and RNA interference of HSP70 on the protein expression of E-cadherin, $\alpha$-SMA, vimentin and collagen I in RPMCs. (A) RPMCs were treated with $25 \mathrm{mM} \mathrm{HG}$ at different time points to induce EMT and the expression of HSP70 was detected by western blotting. (B) Values represent the mean \pm standard error of the mean of three independent experiments performed. $\beta$-actin was used as the loading control. ${ }^{* *} \mathrm{P}<0.01$ vs. $\mathrm{HG} 0 \mathrm{~h}$ group, ${ }^{*} \mathrm{P}<0.05$ vs. HG $0 \mathrm{~h}$ group. (C) qPCR analysis was performed using isolated total RNA from HSP70 mRNA following transfection with a HSP70 overexpression plasmid for different time periods. Each value represents the mean \pm standard error of the mean $(n=3)$. ${ }^{*} \mathrm{P}<0.05$ vs. untreated control. ${ }^{* *} \mathrm{P}<0.01$ vs. control. (D) qPCR analysis was performed using isolated total RNA from HSP70 mRNA following transfection with HSP70-siRNA for different time periods. Each value represents the mean \pm standard error of the mean $(n=3)$. ${ }^{*} \mathrm{P}<0.05$ vs. untreated control. ${ }^{* *} \mathrm{P}<0.01$ vs. control. (E) Control, HSP70-siRNA knockdown and HSP70 overexpression cells were treated with or without $25 \mathrm{mM}$ glucose for $24 \mathrm{~h}$ and the expression of E-cadherin, vimentin, collagen I and $\alpha$-SMA were detected by western blotting. (F) Values represent the mean \pm standard error of the mean of three independent experiments performed. $\beta$-actin was used as the loading control. ( ${ }^{* *} \mathrm{P}<0.01$ vs. control; ${ }^{*} \mathrm{P}<0.01 \mathrm{HG}$ group vs. HG+HSP70 group;. ${ }^{\wedge} \mathrm{P}<0.01 \mathrm{HG}$ group vs. HG+siRNA-HSP70 group). HSP70, heat shock protein 70; $\alpha$-SMA, $\alpha$-smooth muscle actin; siRNA, small interfering RNA; HG, high glucose; RPMCs, rat peritoneal mesothelial cells; EMT, epithelial-to-mesenchymal transition; qPCR, quantitative polymerase chain reaction.

and F). Transiently overexpressing HSP70 significantly reduced the levels of HG-induced EMT, as evidenced by the reduced upregulation of $\alpha$-SMA, vimentin and mesenchymal marker collagen I, and the ameliorated expression of the epithelial protein, E-cadherin. By contrast, siRNA-mediated suppression of HSP70 further exacerbated HG-induced EMT. Notably, siRNA-treated or HSP70 overpression plasmid-treated cultures alone did not induce EMT-mediated changes in the RPMCs.

Effect of HSP70 on Smad pathway in the HG-treated RPMCs. Smad signaling pathways have been reported as important in the EMT (9). To investigate the possible involvement of Smad signaling in the protective effect of HSP70 in RPMCs from HG-induced EMT, control, HSP70-siRNA knockdown and HSP70 overexpression cells were treated with $25 \mathrm{mM}$ glucose, and the expression levels of p-Smad3 and p-Smad4 were detected by western blot analysis. Under controlled conditions, HSP70-siRNA knockdown and HSP70 overexpression did not enhance p-Smad3 and 4 levels compared with those in the control cells. However, following exposure to HG, p-Smad 3 and 4 levels were found to be significantly enhanced in HSP70-siRNA group compared with those in the HG group. Conversely, overexpression of HSP70 significantly inhibited HG-induced phosphorylation of Smad3 and 4 (Fig. 3). Overall, these results suggest that HSP70 may attenuate HG-induced EMT by inhibiting the activated Smad pathway.

HSP70 inhibits ROS and attenuates EMT of peritoneal mesothelial cells. It has been shown that oxidant/antioxidant imbalance can activate multiple molecular pathways that culminate in the induction of EMT in target cells: ROS could activate Smad signaling molecules, which are crucial for EMT (10). Thus, the HG-induced ROS levels in different treatment groups were detected. The cells were stained with DCF-DA to detect the intracellular ROS production. To further analyze the involvement of ROS in the course of EMT, the effect of HG in the presence of the antioxidant, $\mathrm{N}$-acetylcysteine (NAC) was assessed. As shown by the results of Fig. 4A, HG increased the 
A

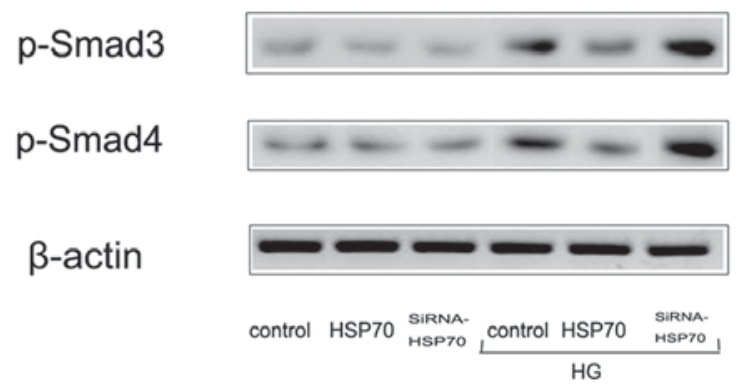

B

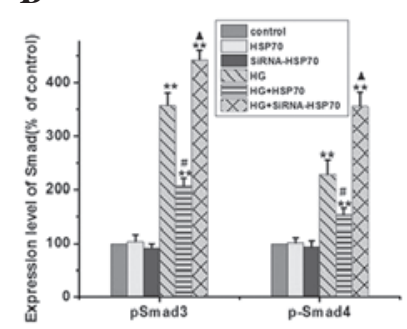

Figure 3. Effect of HSP70 on the Smad pathway in HG-treated RPMCs. (A) Control, HSP70-siRNA knockdown and HSP70 overexpression cells were treated with or without $25 \mathrm{mM}$ glucose for $24 \mathrm{~h}$ and the expression of p-Smad3 and p-Smad4 was detected by western blotting. (B) Values represent the mean \pm standard error of the mean of three independent experiments performed. $\beta$-actin was used as the loading control. ${ }^{(* *} \mathrm{P}<0.01 \mathrm{vs}$. control; ${ }^{*} \mathrm{P}<0.01 \mathrm{HG}$ group vs. HG+HSP70 group; ${ }^{`} \mathrm{P}<0.01 \mathrm{HG}$ group vs. HG+siRNA-HSP70 group). HSP70, heat shock protein 70; siRNA, small interfering RNA; HG, high glucose; RPMCs, rat peritoneal mesothelial cells.

A

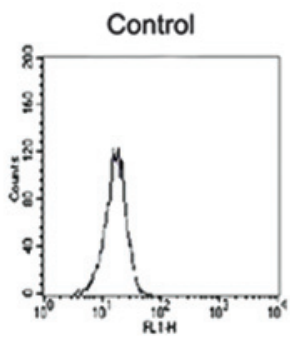

B

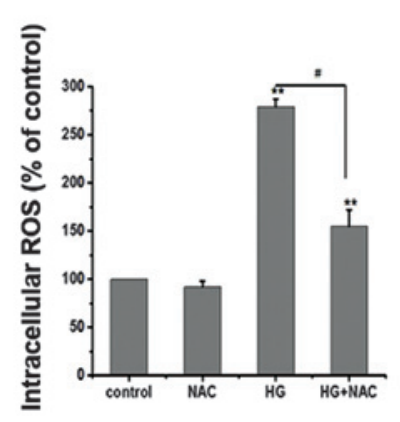

HG

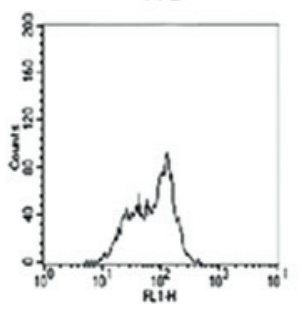

C
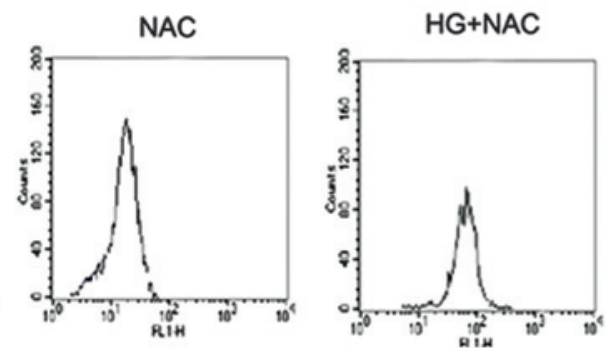

D

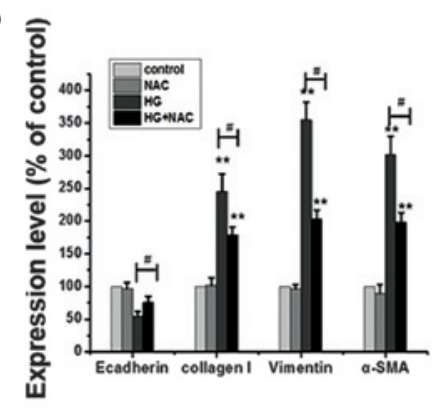

$\mathbf{E}$

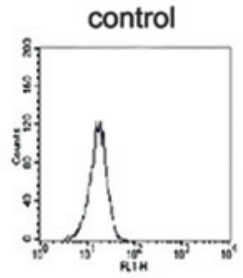

HG

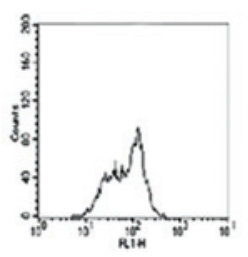

HSP70

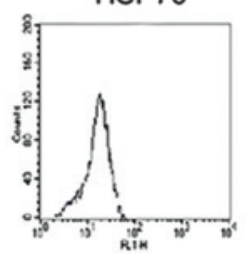

HG+HSP70

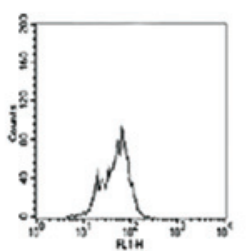

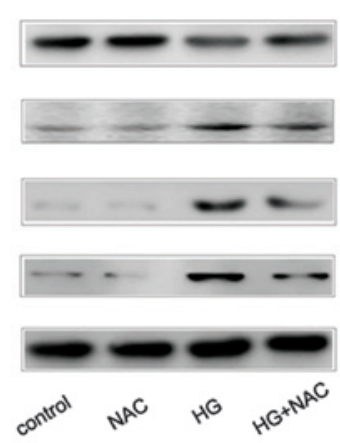

SiRNA-HSP70

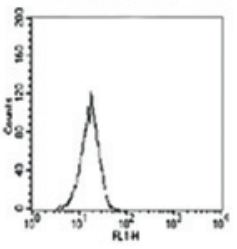

HG+SiRNA-HSP70

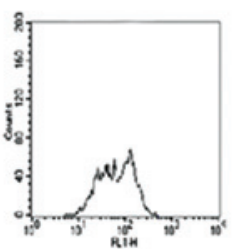

F

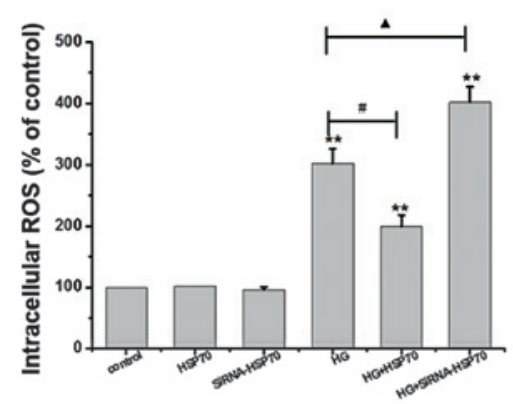

Figure 4. HSP70 reduces HG-induced ROS and inhibits HG-induced EMT in RPMCs. (A and B) Cells were exposed to $25 \mathrm{mM}$ glucose for $24 \mathrm{~h}$, or a combined treatment of $25 \mathrm{mM}$ glucose and $10 \mathrm{mmol} / 1 \mathrm{NAC}$ for $24 \mathrm{~h}$, and the cells were stained with DCF-DA to detect the intracellular ROS production. ${ }^{* * *} \mathrm{P}<0.01 \mathrm{vs}$. control; " $\mathrm{P}<0.01 \mathrm{HG}$ group vs. HG+NAC group. (C and D) Cells were treated as previously described, and the expression of E-cadherin, vimentin, collagen I and $\alpha$-SMA were detected by western blotting. Values represent the mean \pm standard error of the mean of three independent experiments performed. $\beta$-actin was used as the loading control. ${ }^{* *} \mathrm{P}<0.01$ vs. control; ${ }^{*} \mathrm{P}<0.01 \mathrm{HG}$ group vs. HG+NAC group. (E and F) Control, HSP70-siRNA knockdown and HSP70 overexpression cells were treated with or without $25 \mathrm{mM}$ glucose for $24 \mathrm{~h}$ and the cells were stained with DCF-DA to detect the intracellular ROS production. ${ }^{* *} \mathrm{P}<0.01$ vs. control; ${ }^{*} \mathrm{P}<0.01 \mathrm{HG}$ group vs. HG+HSP70 group; ${ }^{\mathrm{P}} \mathrm{P}<0.01 \mathrm{HG}$ group vs. HG+siRNA-HSP70 group. HG, high glucose; NAC, N-acetylcysteine; HSP70, heat shock protein 70; ROS, reactive oxygen species; $\alpha$-SMA, $\alpha$-smooth muscle actin; siRNA, small interfering RNA; EMT, epithelial-to-mesenchymal transition; RPMCs, rat peritoneal mesothelial cells; DCF-DA, dihydrodichlorofluorescein diacetate. 
levels of intracellular ROS production compared with those in the control cells. However, HG-induced ROS production was significantly blocked by pretreatment of cells with NAC (Fig. 4A and B). The western blot analysis results demonstrated that $\mathrm{HG}$ reduced the expression levels of the protein E-cadherin and increased the expression levels of the proteins vimentin, collagen I and $\alpha$-SMA, compared with those in the control cells. HG-mediated changes in the expression levels of the EMT proteins were abolished by NAC (Fig. 4C and D). These results indicate that ROS generation induced by HG has an important role in EMT of peritoneal mesothelial cells. Subsequently, the role of HSP70 in HG-induced ROS generation was investigated. Under controlled conditions, HSP70-siRNA knockdown and HSP70 overexpression did not markedly change the levels of intracellular ROS production compared with those in the control cells. However, following exposure to $\mathrm{HG}$, the ROS levels were found to be significantly enhanced in the HSP70-siRNA group compared with those in the HG group. Conversely, overexpression of HSP70 significantly inhibited HG-induced ROS production and HG-induced EMT (Fig. 4E and F). Overall, these results suggest that HSP70 may attenuate HG-induced EMT by inhibiting ROS production.

\section{Discussion}

By the end of 2005, the number of patients with PD displayed a rapid growth trend (10). Globally, the number of patient with PD was $\sim 160,000$ in 2012 , accounting for $11 \%$ of the total number of patients receiving dialysis (11). However, the ultrafiltration failure resulting from peritoneal fibrosis is one of the main complications that occur after long periods of PD, and compels patients to withdraw from PD. A previous study implicates the appearance of the EMT as the main point in the early pathogenesis of the development and progression of peritoneal fibrosis (12). Another study showed that HG could induce EMT in vivo (2). Therefore, the present study was conducted to identify the factors that reduce or inhibit EMT. In this study, it was found that HG could induce EMT and lead to increased EMT events in peritoneal mesothelial cells.

HSP70, as a ubiquitously expressed protein, is upregulated by variable stresses, including heat, oxidative stress, anticancer chemotherapy and chemical injury in the cells (13). Thus, we hypothesized that HSP70 may be involved in HG-induced EMT. In the present study, it was demonstrated that HG lead to enhanced levels of HSP70, with induction sustained upon HG stimulation compared with those of the control. To determine the role of HSP70, the effects of HSP70 were studied by modulating HSP70 expression through siRNA knockdown and overexpression. This study found that overexpressing HSP70 attenuated $\mathrm{HG}$-induced upregulation of collagen I and $\alpha$-SMA and ameliorated E-cadherin expression in the RPMCs, while siRNA-mediated suppression of HSP70 further exacerbated HG-induced EMT.

It is well-established that EMT is stimulated through the Smad signaling pathway and inhibition of Smad signaling is a central mechanism in the prevention of peritoneal fibrosis $(14,15)$. In order to further confirm the effect of HSP70 on Smad pathways in HG-induced EMT in RPMCs, the present study investigated the expression of HG-induced phosphory- lated Smad3 and Smad4 by HSP70 overexpression. It was found that overexpressed HSP70 significantly inhibited HG-induced phosphorylation of Smad3 and 4. These results suggest that HSP70 may reduce EMT by inhibiting the activation of HG-induced Smad pathways.

ROS have been widely considered as critical cellular signaling molecules involved in various biological processes, including cell growth, differentiation, proliferation, apoptosis and angiogenesis $(16,17)$. The homeostasis of ROS is critical to maintain normal biological processes. A previous study has shown that ROS is important in the regulation of EMT (18). In the present study, ROS levels were found to be significantly enhanced in the HSP70-siRNA group exposed to $\mathrm{HG}$, compared with those of the HG group. Conversely, overexpression of HSP70 significantly inhibited HG-induced ROS production. High concentrations of glucose may alter the intracellular redox state and this effect is mediated by activation of a phosphokinase $\mathrm{C}$ (polyol pathway) or by altering the NADH/NAD ratio responsible for pseudohypoxia conditions (19). In the present study, ROS expression induced by HG promoted EMT in RPMCs. A number of studies reported that HSP70 has an antioxidative effect $(20,21)$. In the present study, it was found that overexpression of HSP70 suppressed HG-induced ROS upregulation, as demonstrated by flow cytometric analysis. We consider that HSP70 could be identified as a promising therapeutic target of peritoneal fibrosis, as selective blockade of HSP70 exacerbated the EMT-mediated changes.

Taken together, the data of the present study demonstrate that HSP70 is critically involved in the regulation of EMT induced by $\mathrm{HG}$ and contributes to reduced EMT events in peritoneal mesothelial cells. Therefore, the use of HSP70 upregulation, potentially achieved via Smad inhibition, to reduce ROS release and improve clinical efficacy may represent a novel therapeutic strategy for PD patients with peritoneal fibrosis.

\section{References}

1. Jager KJ, van Dijk PC, Dekker FW, Stengel B, Simpson K and Briggs JD; ERA-EDTA Registry Committee: The epidemic of aging in renal replacement therapy: an update on elderly patients and their outcomes. Clin Nephrol 60: 352-360, 2003.

2. Lee YJ and Han HJ: Troglitazone ameliorates high glucose-induced EMT and dysfunction of SGLTs through PI3K/Akt, GSK-3 $\beta$, Snail1, and $\beta$-catenin in renal proximal tubule cells. Am J Physiol Renal Physiol 5: F1263-F1275, 2010.

3. Alisson-Silva F, Freire-de-Lima L, Donadio JL, Lucena MC Penha L, Sá-Diniz JN, Dias WB and Todeschini AR: Increase of O-glycosylated oncofetal fibronectin in high glucose-induced epithelial-mesenchymal transition of cultured human epithelial cells. PLoS One 8: e60471, 2013.

4. Naber HP, Drabsch Y, Snaar-Jagalska BE, ten Dijke P and van Laar T: Snail and Slug, key regulators of TGF- $\beta$-induced EMT, are sufficient for the induction of single-cell invasion. Biochem Biophys Res Commun 435: 58-63, 2013.

5. Lu X and Kakkar V: The role of heat shock protein (HSP) in atherosclerosis: Pathophysiology and clinical opportunities. Curr Med Chem 17: 957-973, 2010.

6. Mayer MP and Bukau B: Hsp70 chaperones: cellular functions and molecular mechanism. Cell Mol Life Sci 62: 670-684, 2005.

7. Chatterjee M, Andrulis M, Stühmer T, Müller E, Hofmann C, Steinbrunn T, Heimberger T, Schraud H, Kressmann S, Einsele $\mathrm{H}$ and Bargou RC: The PI3K/Akt signaling pathway regulates the expression of Hsp70, which critically contributes to Hsp90-chaperone function and tumor cell survival in multiple myeloma. Haematologica 98: 1132-1141, 2013. 
8. Mikuriya T, Sugahara K, Takemoto T, Tanaka K, Takeno K, Shimogori H, Nakai A and Yamashita H: Geranylgeranylacetone, a heat shock protein inducer, prevents acoustic injury in the guinea pig. Brain Res 1065: 107-114, 2005.

9. Margetts PJ, Bonniaud P, Liu L, Hoff CM, Holmes CJ, West-Mays JA and Kelly MM: Transient overexpression of TGF- $\{$ beta $\} 1$ induces epithelial mesenchymal transition in the rodent peritoneum. J Am Soc Nephrol 16: 425-436, 2005.

10. Rhyu DY, Yang Y, Ha H, et al: Role of reactive oxygen species in TGF-beta1-induced mitogen-activated protein kinase activation and epithelial-mesenchymal transition in renal tubular epithelial cells. J Am Soc Nephrol 16: 667-675, 2005.

11. Grassmann A, Gioberge S, Moeller S and Brown G: End-stage renal disease: global demographics in 2005 and observed trends. Artif Organs 30: 895-897, 2006.

12. Fang CC, Huang JW, Shyu RS, Yen CJ, Shiao CH, Chiang CK Hu RH and Tsai TJ: Fibrin-induced epithelial-to-mesenchymal transition of peritoneal mesothelial cells as a mechanism of peritoneal fibrosis: effects of pentoxifylline. PLoS One 7: e44765, 2012.

13. Grune T, Catalgol B, Licht A, Ermak G, Pickering AM, Ngo JK and Davies KJ: HSP70 mediates dissociation and reassociation of the $26 \mathrm{~S}$ proteasome during adaptation to oxidative stress. Free Radic Biol Med 51: 1355-1364, 2011.

14. Yao Q, Pawlaczyk K, Ayala ER, Styszynski A, Breborowicz A Heimburger O, Qian JQ, Stenvinkel P, Lindholm B and Axelsson J: The role of the TGF/Smad signaling pathway in peritoneal fibrosis induced by peritoneal dialysis solutions. Nephron Exp Nephrol 109: e71-e78, 2008.
15. Neil JR, Johnson KM, Nemenoff RA and Schiemann WP: Cox-2 inactivates Smad signaling and enhances EMT stimulated by TGF-beta through a PGE2-dependent mechanisms. Carcinogenesis 29: 2227-2235, 2008.

16. Loor G, Kondapalli J, Schriewer JM, Chandel NS Vanden Hoek TL and Schumacker PT: Menadione triggers cell death through ROS-dependent mechanisms involving PARP activation without requiring apoptosis. Free Radic Biol Med 49: 1925-1936, 2010.

17. Chen Z, Pittman EF, Romaguera J, Fayad L, Wang M, Neelapu SS, McLaughlin P, Kwak L and McCarty N: Nuclear translocation of B-cell-specific transcription factor, BACH2, modulates ROS mediated cytotoxic responses in mantle cell lymphoma. PLoS One 8: e69126, 2013.

18. Hu Y, He K, Wang D, Yuan X, Liu Y, Ji H and Song J: TMEPAI regulates EMT in lung cancer cells by modulating the ROS and IRS-1 signaling pathways. Carcinogenesis 34: 1764-1772, 2013.

19. Henningsen $C$, Zahner $G$ and Thaiss F: High glucose induces type 1 hexokinase gene expression in isolated glomeruli of diabetic rats and in mesangial cells. Nephron Physiol 93: p67-p75, 2003.

20. Di Domenico F, Sultana R, Tiu GF, Scheff NN, Perluigi M, Cini C and Butterfield DA: Protein levels of heat shock proteins 27,32,60, 70, 90 and thioredoxin-1 in amnestic mild cognitive impairment: an investigation on the role of cellular stress response in the progression of Alzheimer disease. Brain Res 1333: 72-81, 2010.

21. Scarpeci TE, Zanor MI and Valle EM: Investigating the role of plant heat shock proteins during oxidative stress. Plant Signal Behav 3: 856-857, 2008. 